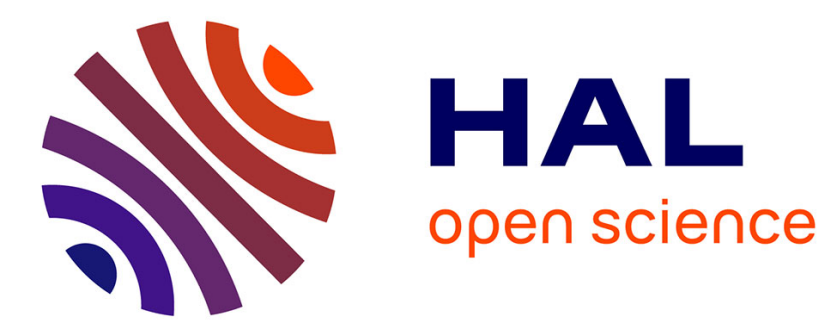

\title{
Measurement Procedures and Uncertainty Evaluation for Electromagnetic Radiated Emissions from Large Power Electrical Machinery
}

Andrea Mariscotti

\section{> To cite this version:}

Andrea Mariscotti. Measurement Procedures and Uncertainty Evaluation for Electromagnetic Radiated Emissions from Large Power Electrical Machinery. IEEE Transactions on Instrumentation and Measurement, 2007, 56 (6), pp.2452 - 2463. 10.1109/TIM.2007.908351 . hal-02970374

\section{HAL Id: hal-02970374 \\ https://hal.science/hal-02970374}

Submitted on 18 Oct 2020

HAL is a multi-disciplinary open access archive for the deposit and dissemination of scientific research documents, whether they are published or not. The documents may come from teaching and research institutions in France or abroad, or from public or private research centers.
L'archive ouverte pluridisciplinaire HAL, est destinée au dépôt et à la diffusion de documents scientifiques de niveau recherche, publiés ou non, émanant des établissements d'enseignement et de recherche français ou étrangers, des laboratoires publics ou privés. 


\title{
Measurement Procedures and Uncertainty Evaluation for Electromagnetic Radiated Emissions from Large Power Electrical Machinery
}

\author{
A. Mariscotti \\ Dipartimento di Ingegneria Elettrica, Via dell’Opera Pia, 11A - 16145 Genova - Italy
}

\begin{abstract}
A. Mariscotti, "Measurement Procedures and Uncertainty Evaluation for Electromagnetic Radiated Emissions from Large Power Electrical Machinery," IEEE Transactions on Instrumentation and Measurement, Vol. 56, No. 6, Dec. 2007, pp. 2452-2463. doi: 10.1109/TIM.2007.908351
\end{abstract}

\begin{abstract}
Methods of measurement and data analysis are considered for the electromagnetic (e.m.) emissions of large power rotating machinery (in this paper a synchronous generator is considered as working example). The data recorded during two measurement campaigns are used to proof the analysis methods and to better describe the measurement procedures. Several important factors are considered: the reactive behavior of the field, site attenuation and antenna factor determination, the physical dimensions of the source of emissions and its impact on correction factor uncertainty, the e.m. environment and the ambient e.m. noise, practical factors (including safety distances, reproducible test conditions, etc.) that limit the choice of the measuring position and distance and determine measurement accuracy.
\end{abstract}

\section{Keywords}

Electric machines, Electromagnetic measurements, Electromagnetic compatibility, Antenna measurements, Electric fields, Magnetic fields

\section{Introduction}

It is now commonly believed [1] that electrical drive systems must be CE marked also for 89/336 EMC directive [2], which implies the application of the product standard EN 61800-3 [3] or the generic standards EN 61000-6-2 [4] and EN 61000-6-3 [5]. A comprehensive discussion of EMC issues for electrical drive systems may be found in [6].

Electrical drive systems are "relevant apparatus", that is "they must have a dedicated purpose requiring no further modification by the end user”. This definition may be easily extended to (large power) rotating machinery, which is marketed as it is without any significant further modification for various applications consisting of power generation itself and electric drives without a static converter, but only connection to the mains network (e.g. pumps, cranes, lifters etc.). 
Only recently electrical rotating machinery has been considered as a source of e.m. emissions other than the magnetic field at the supply frequency and its harmonics. Few contributions may be found in the literature concerning the measurement of e.m. emissions, all concentrated in the last five years [7][9]. In the last years limits on the e.m. emissions have been proposed to ensure the electromagnetic compatibility (EMC) of electrical rotating machinery. The first contribution to the definition of emission limits was the amendment A2 to EN 60034-1 [10], where the problem of e.m. emissions from rotating machinery is addressed with particular attention to commutator machines. After, the standard EN 60034-1 has been issued as version 2 [11], where some measurement guidelines are added to the emission limits. The limits are still specified for the high frequency range (above 30 $\mathrm{MHz}$ ); the low frequency range (between $9 \mathrm{kHz}$ and $30 \mathrm{MHz}$ ) is considered only for conducted emissions. It may be expected in general that EMC problems - if any - are more likely to occur in the low and medium frequency range (below few megahertz), where the rotating machine is judged to be a significant source of radiated emissions and where effective shielding of magnetic emissions cannot be obtained by means of thin metallic sheaths or the enclosure itself. In section 12 of the standard EN 600034-1 [11] it is specified that "the requirement of section 12 ("Electromagnetic Compatibility") do not apply to machines when the EMC performance is significantly affected by the final enclosure and assembly”. Furthermore, the EMC section of the EN 60034-1 standard seems to be derived from other EMC standards [12], which are not strictly applicable to large power rotating machinery and within its typical e.m. environment. The reasons for this will be explained in the next section. These two considerations indicate clearly that the variations between different test sites might compromise measurement reproducibility (as stated in [13] for conducted emission tests). Applicable standards give technicians several degrees of freedom in antenna positioning, treatment of transient phenomena and of external disturbance and setup of machinery operating conditions, thereby introducing additional sources of systematic error and uncertainty. Setup and environmental uncertainty may be

larger than that related directly to measuring instruments and accessories, which are usually well defined and documented. The measurement system consists of one or more antennas, signal conditioning elements like attenuators, filters and preamplifiers, a frequency domain measuring instrument (like a spectrum analyzer or measuring receiver) and data recording equipment. These elements are connected with cables, which can have a major impact on the accuracy of test results, especially at higher frequencies [14].

The goal of this paper is the definition of adequate measurement procedures and the evaluation of error sources and measurement uncertainty.

\section{Machine under test and electromagnetic environment}

The assessment of emission level must be done by means of direct measurement, which should be performed in a well defined environment in reproducible conditions, as it is stated in the EMC 
standards for e.m. emissions [3][10][11]. This is a reasonable task for a low power device or equipment, that may be easily tested in an Open Area Test Site or in a anechoic chamber, but not for a large power machine of very large size, that requires specific placement, auxiliary equipment and availability of a large power network.

For large power rotating machinery some practical problems, as identified in [8], must be considered.

The source of emissions is quite extended in space, both in the direction of emissions and in the orthogonal plane, since the largest dimension of the machine under test is larger than the recommended measuring distance [11]. It may be difficult to perform the measurement at the recommended measuring distance, so that the measuring distance must be determined on the basis of the minimal acceptable signal level (depending on the desired signal-to-noise ratio) and of other safety and practical constraints (minimal safety distance, presence of inspection holes, metallic structures). Similar problems occur for large military equipment and the procedure indicated in Method RE102 of the MIL std. 462D [15] is simply that of placing $P$ (equally spaced) measuring positions $1 \mathrm{~m}$ away from the EUT (Equipment Under Test) boundary, with $P=\lceil X / 3\rceil$ (round up to the next integer), where $X$ is the largest EUT dimension. The EN 60034-1 standard [11] indicate a measuring distance of $10 \mathrm{~m}$ or $30 \mathrm{~m}$ for machines operating without and with brushes respectively; a $3 \mathrm{~m}$ distance may be used if the limits are increased by $10 \mathrm{~dB}$ or $20 \mathrm{~dB}$ respectively. Yet, the far field assumption behind the $10 \mathrm{~dB}$ or $20 \mathrm{~dB}$ increase holds only for the high frequency range above $30 \mathrm{MHz}$ covered by the standard and not for the lower frequency range, where the most significant emissions are expected.

Large power rotating machines require several auxiliary systems (lubricating and cooling pumps, static converter for the excitation system and other auxiliary circuits, and also a driving motor for the control of rotation speed of generators) and the e.m. noise generated by the machine itself cannot be easily identified and separated.

The EN 61800-3 standard [3] (concerning EMC requirements and test procedures for adjustable speed drives) states at section 6.3.2.3 that "if the ambient noise (without operation of the Power Drive System which is supposed to be the emitter) is over the limits, the supposed emitting PDS is only considered to fail if a characteristic set of emitted frequencies can be recognised and is over the measured ambient noise”. This is true and applicable if the source of emissions is narrowband (and rotating machinery generally is not); furthermore, noise from external sources or auxiliary systems may appear as narrowband noise, and it may be erroneously attributed to the machinery under test (see Fig. 1). In [16] DC motors are indicated as a source of broadband noise due to commutation arcing: it must be underlined that very low power DC motors were referred to and that focus was on high frequency electric field emissions; for large power motors emphasis is on magnetic field emissions on a lower frequency range [8]. In [20][21] it is suggested to subtract the electric field strength of the radio external sources from the measured e.m. field value to obtain the exact value of machine 
emissions. Results have been tested for laboratory produced stationary ambient noise and cancellation was almost perfect [21]; the author questions if the cancellation is satisfactory also with true ambient noise signals and he preliminarily concludes that cancellation is not satisfactory and that can be improved if quasi-peak detector is used instead of peak detector. Direct cancellation may be applicable to some extent for broadcast radio stations, since the emitted power may be assumed nearly constant during night and day, but not for other narrowband radiofrequency sources (like radio-amateur or military systems): the amplitude of the e.m. field they produce is not constant, since they may be switched on and off and their transmission power may be modulated, so that they may result in variable amplitude components over the different recordings and may be confused with machine emissions; in this case the knowledge of the authorized transmission frequencies is of fundamental importance for external sources recognition and exclusion of the polluted bands. So, when the average value of several successive recordings of the ambient noise is used, as the best estimate of the $\mathrm{E}$ or $\mathrm{H}$ field strength of external sources, the estimated uncertainty (estimated as the sample dispersion, see section 4.2) gives importance information on the amplitude distribution. Two examples of radio sources of the first and second type are given in Fig. 2 (test room noise) for the $1.3 \mathrm{MHz}$ component and the $26-27 \mathrm{MHz}$ frequency band respectively (see also section 4.2 for uncertainty calculation).

The minimum margin $m$ between the ambient noise $N$ and the limits (or machine emissions) $S$, identified above as signal-to-noise ratio $S / N$, is often set to $m=6 \mathrm{~dB}$ or even $m=10 \mathrm{~dB}$; the impact on the total field strength $S+N$ (under the assumption of rms summation rule, that is noise and signal are uncorrelated) is an increase of only about $1.0 \mathrm{~dB}$ and $0.4 \mathrm{~dB}$ respectively above $S$. So, the margin $m$ is adequate for the correct evaluation of machine emissions and limits crossing, only if $N$ is a correct estimate of the noise level for any measurement at any time. Again, a careful and complete characterization of the noise produced by external sources is needed; $N$ may be determined as the maximum noise level, by using the envelope of maxima as a worst case, or as the level below which the noise is for $95 \%$ of the time, by using the estimated uncertainty, for highly variable intermittent external sources.

The test site is far from being similar to an Open Area Test Site (as described by CISPR 16 [17] or ANSI C63.4 [26]), since the ground is clearly imperfect and because of the presence of reflecting and scattering surfaces inside the Obstruction Free Area (OFA), due to the presence of concrete blocks, steel rods, pipes, etc. The assumption under the OFA elliptical shape indicated by the standards [12][17] is that the EUT is placed on a turntable; this is clearly not possible with machines of considerable weight and size, so that the recommended OFA is a circular area of large radius $R$, compared to the EUT to antenna distance $d$. In [20] the $R / d$ ratio is set to 3.16 to ensure that the path of any reflected ray is 3.16 longer than the path of the direct ray between the EUT and the antenna, so that by the path loss formula the attenuation is $10 \mathrm{~dB}$ minimum, under the assumption of far field decay of the e.m. field. 
Test conditions are sometimes critical and machine operating conditions cannot be held constant for long time intervals, like for the short circuit test; in this case the critical aspects are first, the sweeping time of the RF receiver or the spectrum analyzer used to measure and to record the emissions and second, the synchronization of the recording with the transient event.

A similar problem is considered in the EN 50121-2 standard [18] for the measurement of train e.m. emissions. Trains are a moving source and emissions are related to specific operating conditions of onboard apparatus, so a specific statistical criterion (peak 80/80) is indicated: the emission value which is compared with the limit curve is computed as "the peak value which would not be exceeded on $80 \%$ of train passages, with $80 \%$ certainty". In this standard we read "since in general only a small number $n$ of tests can be carried out, the non-central t-distribution method mentioned in EN 55013 standard [19] shall be used to find the required exceedance and certainty values. This statement recognize that tests are difficult to arrange and it allows a relatively small number of tests to be used”.

Moreover, it is in general difficult to fully monitor and/or record all machine variables (mainly machine windings current and voltage), since large amplitude and wide bandwidth are generally opposite requirements for the current and voltage sensors. So, the relationship of measured emissions with machine variables cannot be defined for an extended frequency range, but only for the low frequency portion, or most often using only rms values.

Since the measuring conditions cannot be fully controlled (switching on and off of cooling and lubricating pumps, moving of bridge cranes, uncontrollable remote sources, etc.), several recordings of e.m. emissions must be performed and statistically post-processed to estimate setup uncertainty and to extract a usable emissions spectrum.

The antennas chosen for the measurements are (active and passive) electric and magnetic antennas. For the choice of the specific antenna, the extension of the frequency range and the required sensitivity and accuracy must be considered. The measurements shown in the reference cases were performed using two active antennas (one EMCO 6502 loop antenna and one EMCO 3301 rod antenna for H and E field measurement over the [9 kHz - $30 \mathrm{MHz}$ ] frequency range) and one passive antenna (one PMM biconical antenna for E field measurement over the $[30 \mathrm{MHz}-200 \mathrm{MHz}]$ frequency range). Several bandwidths values were set on the receiver/spectrum analyzer: $200 \mathrm{~Hz}$ or $1 \mathrm{kHz}$ for [9 kHz - $150 \mathrm{kHz}$ ] frequency range; $1 \mathrm{kHz}$ and $9 \mathrm{kHz}$ or $10 \mathrm{kHz}$ for the [150 kHz - $30 \mathrm{MHz}$ ] frequency range; $100 \mathrm{kHz}$ or $120 \mathrm{kHz}$ for the [30 MHz - $200 \mathrm{MHz}$ ] frequency range. (Brand names shown here are only informative and there is no explicit or implicit endorsement)

Loop antenna is a directional antenna with a typical main lobe angle of 40 to 60 degrees (from the inspection of the radiation pattern): a series measurements is necessary at the same location at every (approximately) 45 degrees of orientation, in order to get an omnidirectional picture of the field 
amplitude on a given plane; an additional reading is necessary to measure the amplitude in the orthogonal plane.

Rod antenna is composed of the rod element and the ground plane, so that the correct position of the antenna with respect to the ground is the vertical position. Then, the vertical component of the electric field is the output of this device, while the components parallel to the ground cannot be measured; to this aim other kinds of electric field antennas (e.g. dipole antennas) must be used, if the horizontal component of the E field must be measured.

Biconical antenna is a large bandwidth dipole (the radiation pattern is a toroidal shape with a omnidirectional pattern in the plane orthogonal to antenna axis and a main lobe angle of about $60^{\circ}$ in the plane containing antenna axis), so that three separate measurements are necessary for a complete $\mathrm{E}$ field characterization (three E field components): usually the most important component is the vertical one (in relation with first, the typical propagation of $\mathrm{E}$ field from remote sources and second, the absence of significant sources with specific polarization/orientation inside the machine under test), even if, we will see later in section 3.3, the horizontal component is preferred in some cases for site attenuation measurements with reduced uncertainty.

Some reference cases are used to show and verify the measurement procedure.

Several synchronous generators have been tested in the Generator Test Room (GTR) at Ansaldo Energia, Genova, Italy. The GTR map is reported in Fig. 3, where the four letters A, B, C and D indicate the four reinforced concrete platforms (with connections to supply and auxiliaries), where the machines are normally set-up for tests; the three areas where the preliminary noise measurements were performed are identified as Z1, Z2 and Z3. The position of one of the generators during tests is shown in Fig. 4, where the two selected measurement positions are indicated with B1 and B2; the selection process was based on: inspection of the test room noise spectra for different zones, sample acquisitions to check for the best signal-to-noise ratio and line-of-sight access to the rotor and stator windings end, practical considerations concerning safety and obstruction free areas.

The electrical characteristics of the tested generators are reported in Table I. Three test conditions are considered: 1) the generator is only moved by the driving dc motor (dc motor emissions are considered as ambient noise in this case); 2) the generator is tested in no-load condition; 3) the generator is tested in a semi-permanent short circuit condition. A picture of the generator G1 is shown in Fig. 5.

\section{Uncertainty evaluation}

Several sources of uncertainty are defined and considered in the following. From a general point of view, measurement uncertainty may be related to systematic effects and random effects. Systematic effects concern equipment setup and measurement misoperations and they may be recovered with 
adequate countermeasures: auto-calibration for spectrum analyzers and receivers; offset correction; check of instrument setup (for instance gain setting, attenuators on and off, post-processing filters, like quasi-peak/average detector, video filter, etc); check of all parameter data taken as a-priori given data (like EUT-antenna distance, site dimensions, objects location, antenna height and orientation); the application of these countermeasures leave residual uncertainty. In the following random errors and uncertainty will be considered. Uncertainty evaluation is performed with two methods [20][22].

Type A, where the uncertainty is calculated from the statistics of a series of repeated observations $x_{\mathrm{i}}$ as the standard deviation $u\left(x_{\mathrm{i}}\right)$ of the mean of the observations; a Gaussian distribution is assumed for the determination from the sample standard deviation and non-central t-distribution method (mentioned in the EN 55013 standard [19]) is used to cope with the reduced number of samples available in practical EMC measurements.

Type B, where the uncertainty is calculated on the basis of assumed (or known) distributions for system components and instrumentation, which fall within one of the following three distributions: Gaussian (normal), rectangular, U-shaped [23]. A rectangular distribution is suited for instruments where accuracy is simply stated as $\pm x \%$ or $\pm x \mathrm{~dB}$, with equal probability for all error values within the interval; standard uncertainty is given by $u=x / \sqrt{3}$. In EMC measurements a U-shaped distribution often exist for VSWR caused uncertainties; depending on the kind of resonance between the transmission line and the generator/load connected at the two ends, the mismatch uncertainty is expressed in terms of $u=20 \log _{10}\left(1 \pm\left|\Gamma_{\mathrm{G}}\right|\left|\Gamma_{\mathrm{L}}\right|\right) / \sqrt{2}$ (with $\Gamma_{\mathrm{G}}$ and $\Gamma_{\mathrm{L}}$ the reflection coefficients at generator and load connectors) and it is evident that the uncertainty interval is not perfectly symmetric around 0. A triangular distribution may be assigned if the distribution of values is bounded between $\pm x$, but the majority of values lie close to the central point (in this case 0 ); standard uncertainty is given by $u=x / \sqrt{6}$. Finally, the normal distribution is assigned to uncertainties derived from multiple contributions, as identified above for Type A evaluation.

\subsection{Instrumentation uncertainty}

The evaluation of the instrumentation uncertainty is performed starting from calibration data and instrument specifications, for all the elements included in the measurement chain: antenna, cable, RF receiver (or spectrum analyzer). The influence of connectors is neglected, given the relatively low frequency values.

\subsubsection{Antenna uncertainty}

For the determination of antenna uncertainty, reference is made to AF (Antenna Factor) and VSWR (Voltage Standing Wave Ratio) specifications; they are determined either from type tests or from individual calibration for all antennas. AF uncertainty is declared by the manufacturer on antenna certificate/specifications and it is reasonably assumed to range between \pm 0.5 and $\pm 1.5 \mathrm{~dB}$, depending 
on frequency and calibration method. Moreover, AF varies in general with the antenna height above ground (not so significantly for the used loop and rod antenna, but much more for dipole antennas, like biconical and log-periodic antennas); this effect is not specified by the manufacturer and it must be in general evaluated by numerical analysis. Active antennas are designed so that AF is almost constant over the entire frequency range, so that the uncertainty related to AF interpolation is very small (nearly $\pm 0.1 \mathrm{~dB}$ ); for high frequency passive antennas a slightly larger uncertainty is indicated in [23] $( \pm 0.25$ $\mathrm{dB})$ or in [14] ( $\pm 0.3 \mathrm{~dB})$. For isotropic antennas an additional uncertainty factor due to anisotropicity must be included, typically equal to $\pm 1.0 \mathrm{~dB}$ or even in particular case (for instance, very small antenna for a very extended frequency range) $\pm 2.0 \mathrm{~dB}$. VSWR at antenna input is in general a function of frequency (larger at frequency band extremes) and antenna height above ground; it may be as high as 2:1 (about 1.5:1 on average) for high frequency passive antennas and less than 1.5:1 for active antennas, thanks to the input impedance matching offered by the antenna input filter and amplifier (with about $\pm 0.25 \mathrm{~dB}$ uncertainty as declared by several manufacturers for individual calibrations). So, VSWR term is responsible for the uncertainty related to the VSWR itself (the input signal is partially reflected back to the source) and for the uncertainty related to the determination of the exact VSWR by the manufacturer.

\subsubsection{Cable uncertainty}

Used cables are RG58 and RG213/214 single shield coaxial cables and manufacturer's data consist of per-unit-length attenuation (as a function of frequency) with often unknown uncertainty and nominal cable characteristic impedance (typ. $50 \Omega$ ); if the cable is assembled and used properly, its uncertainty is so small to be neglected with respect to the uncertainty contributions determined in the following (below $\pm 0.1 \mathrm{~dB}$ as for [24]) and it does not need any in-house accurate calibration procedure. Quite peculiarly in [42], where equipment calibrated against National standards is used, so that the shown uncertainty values are the lowest attainable, the largest source of uncertainty was the 20 m connecting cable and the temperature change due to the sun clearly visible or hidden by clouds during measurements (as high as $0.4 \mathrm{~dB}$ ).

\subsubsection{Receiver/spectrum analyzer uncertainty}

Accurate determination of combined receiver uncertainty is a complex task, since several elements must be considered: receiver noise, input attenuator uncertainty, input VSWR, gain variation for different resolution bandwidths (RBW) filters, spurious response and mixer intermodulation terms, non linearity and scale interpolation errors. Receiver noise floor (below $-100 \mathrm{dBm}$ typ. at $10 \mathrm{kHz}$ RBW, linear function of RBW) is usually well below the normally measured levels. Input attenuator accuracy depends on the frequency band and may be as little as $0.5 / 1.0 \mathrm{~dB}$ below about $1 \mathrm{GHz}$ and slightly higher (2.0/3.0 dB) for larger frequency. Input VSWR depends on the setting of the internal input attenuator, which as any external attenuator improves impedance matching; input VSWR varies 
between 1.2:1 (using the $10 \mathrm{~dB}$ or, usually better, $20 \mathrm{~dB}$ attenuator) and 1.8:1 ( $0 \mathrm{~dB}$ attenuator) below about $1 \mathrm{GHz}$. VSWR may be improved by interposition of an external attenuator with the aim of impedance matching at input port: in this case an additional uncertainty term is represented by the flatness (defined as the peak to peak variation) of the attenuation factor over the entire frequency band, which is typically below a fraction of $\mathrm{dB}$ with respect to nominal attenuation. Gain variation between any two different RBW filters is limited to a fraction of $\mathrm{dB}$. Spurious responses and mixer intermodulation terms (below about $-100 \mathrm{dBm}$ ) are caused by design imperfections of the instrument (they disappear if the input signal is disconnected) and are again well below the normally measured levels. Display dynamic range accuracy depends on either log or linear display mode and typically is below $0.5 \mathrm{~dB}$. These uncertainty estimates are in general a function of the ambient temperature and may be much different for extreme ambient conditions.

The Type B approach to receiver/spectrum analyzer overall combined uncertainty gives a very large pessimistic estimate. Separate and combined uncertainty values are reported in Table II for the used spectrum analyzer [25]. Calculated combined uncertainty is $1.75 \mathrm{~dB}$; the combined uncertainty obtained by direct measurement at few sample frequencies with a reference RF generator (which includes the uncertainty of the RF generator itself and also other uncertainty factors not listed above) is only $1.5 \mathrm{~dB}$. This type of uncertainty is often referred to as "sine wave tests uncertainty" and it is around 1.0-1.5 dB; analogously pulse amplitude wave tests may be determined with abrupt variations

of the amplitude of the applied continuous wave signal. The uncertainty related to the measurement of transient phenomena is a complex task, which includes systematic error terms, due to, among others, wrong choice of the sweeping time and RBW filter.

\subsection{Repeatability uncertainty}

The determination of uncertainty related to repeatability is performed with a Type A approach. $M$ successive recordings are performed for the same measurement setup (machine operating conditions, measurement position, antenna orientation, frequency range, receiver/spectrum analyzer settings). The expanded standard deviation (assuming a confidence level of 95\%) is computed over the entire frequency range and the results are reported in Table III for different tests. Uncertainty calculations have been performed at uniformly selected frequencies from $9 \mathrm{kHz}$ to $200 \mathrm{MHz}$, together with additional frequencies (purposely selected to observe the influence of an evident external noise source and marked by an asterisk). Measurements performed at specific frequency bands used for broadcast commercial and, in particular, amateur radio transmissions should be avoided, since the calculated uncertainty may be very high: it is found that it is 3 to 6 times larger than the maximum uncertainty for all other frequencies, due to the discontinuous behavior of field level. So, for a distribution of field level samples far from the normal distribution, a more robust estimation of standard deviation is the Interquartile Range (the difference between the 75th and the 25th percentiles of the sample). 


\subsection{Test site uncertainty}

The uncertainty due to site imperfection is generally unknown. As extensively described in section 2, the uncertainty of a real Generator Test Room is much larger than that of an Open Area Test Site (which is a highly controlled environment). The OATS uncertainty is determined with reference to variations of several geometric parameters (spacing and flatness of the ground plane mesh, measurement of the distance between antennas and machine under test). Concerning the high frequency range [30 MHz - $1000 \mathrm{MHz}$, the maximum deviation for compliance of the Normalized Site Attenuation (NSA) of an OATS from the theoretical NSA values given in the ANSI C63.4 standard [26] is $\pm 4 \mathrm{~dB}$ : so, the NSA of two compliant OATS may differ by as much as $8 \mathrm{~dB}$ ! This consideration is not pure theory, but it was demonstrated in practice with several Round-Robin tests between OATS and including also Semi-Anechoic Chambers (SAC) and Full Anechoic Chambers (FAC) [27]-[29]: in [27] 10m chambers result in smaller site deviation (approximately $2 \mathrm{~dB}$ below $1 \mathrm{GHz}$, increasing to $3.5 \mathrm{~dB}$ between 2 and $4 \mathrm{GHz}$ ), OATS exhibit larger variations (approximately 1 $\mathrm{dB}$ higher), while $3 \mathrm{~m}$ chambers deviation peak as high as $7 \mathrm{~dB}$, with significant variations; these results are partially confirmed by [29], where 3.5-3.6 dB and 2.5-3.0 dB variations are indicated for SAC and OATS respectively (for horizontal and vertical polarization).

In several publications [28][29] it is underlined that the large part of site uncertainty (the figure itself and the measurement and evaluation method) is given not only by site imperfections, but also by the lack of antenna height scanning and by AF, in the sense of adoption of the correct calibration method for the purposed antenna use and location. It is known that there are several procedures for antenna calibration (ANSI C63.5 and SAE ARP 958 cited in [30], CISPR 16-1 cited in [31]) and the result is most always a simple table, the Antenna Factor: it should be accompanied by a description of the calibration method and related assumptions. Standard ANSI C63.5 now contains several methods for antenna calibration, resulting in geometry-specific AF and free-space AF values [31][32]. Several are the factors that affect free-space assumption and related AF values: 1) mutual coupling between antennas and the ground plane, 2) mutual coupling between antenna themselves, 3) operation in the near field region, 4) non-uniform wave illumination from the transmitting antenna and 5) effects of antenna cable; 6) edge diffraction, even for almost ideal ground planes. ANSI Normalized Site Attenuation (NSA) procedure [25] relies mainly upon tuned dipoles, which despite their simple geometry are far from being electrically small, so that mutual coupling effects are an issue, especially in the lowest part of the frequency range; the ANSI model assumed a far-field radiation pattern of a point-dipole, used in a condition where this assumption is no longer valid [33]; other errors related to biconical and log-periodic antennas have been discovered later in [34][35]. Apart from which procedure to use for site validation and for AF determination, it is stressed that the above described uncertainty factors are all additive and most often partially not included in the antenna calibration certificate and, as a consequence, in site evaluation. If NSA evaluation is done by means of a biconical antenna and related $\mathrm{AF}$ in the $[30 \mathrm{MHz}-300 \mathrm{MHz}$ ] frequency range, the computed corrective term 
$\Delta \mathrm{AF}_{\mathrm{TOT}}$ (accounting not only for the mutual impedance correction, but in general for 1) through 4) sources of uncertainty identified above, as done per ANSI C63.4 [26][32] with reference dipoles) is as high as $2.2 \mathrm{~dB}$ in the [30 MHz - $200 \mathrm{MHz}$ ] interval and up to $4.6 \mathrm{~dB}$ if the interval extends up to 300 $\mathrm{MHz}$, which represent, if not corrected, the systematic error for NSA evaluation in this frequency interval. Smith [39] identifies that mutual impedance correction is needed for closely spaced horizontally polarized antennas below approximately $100 \mathrm{MHz}$; in his paper (Section II-F) he gives simple formulae for the calculation of the corrective term for resonant dipole: the values for a simple geometry stay within +2.9 and -2.4 for the $[30 \mathrm{MHz}-200 \mathrm{MHz}$ ] frequency interval. When mutual coupling correction is applied, calculated and measured site attenuation agree within $2 \mathrm{~dB}$ below 200 $\mathrm{MHz}$ and approximately $3.5 \mathrm{~dB}$ if the interval is extended up to $1 \mathrm{GHz}$. The last factor 6) is to be accounted for not only in "real-world test sites" but it is also common to OATS: in [42] (in this case the NPL, UK OATS is considered) edge diffraction for two VP biconical antennas over a $60 \mathrm{~m} \times 30 \mathrm{~m}$ steel plate ground plane produces a NSA ripple above $160 \mathrm{MHz}$ as large as $\pm 0.3 \mathrm{~dB}$ with the soil at the perimeter dry, while only $\pm 0.1 \mathrm{~dB}$ with wet soil (edge currents are better grounded).

Two separate effects are under the term "effects of (e.m. interaction with) antenna cable", as identified in [40]: the cable itself is a scatterer and, more significantly, induced currents on the outer shield may leak into the antenna with an imperfect (unbalanced) balun. Since cable goes down vertically from antenna connector to the floor, scattering effects influence VP antenna response $( \pm 3 \mathrm{~dB}$ on average for a $\pm 25 \mathrm{~cm}$ swing back and forth of the cable arrival point on the floor) and only marginally the HP antenna response (below $\pm 0.2 \mathrm{~dB}$ approximately) [40]. It is underlined that in [42], Table 3 notes, "the feed cable extends horizontally behind the antenna for at least $2 \mathrm{~m}$ before dropping vertically", so that the declared uncertainty is only $0.05 \mathrm{~dB}$, including the effect of masts. For the second effect we may say that it is directly proportional to the balun unbalance percentage: from the simulations in [40], where the same cable swing as above was also applied, it is observed from comparison with the results for perfect balun that the increase in the spread of HP and VP response is roughly equal to the applied balun unbalance but only above $200 \mathrm{MHz}$. It must be underlined that results below $200 \mathrm{MHz}$ seem to exhibit an opposite behavior, with slightly better response for unbalance balun: the evaluation of balun performances is not trivial and straightforward and it is underlined that usually the behavior of the balun accompanying a dipole or biconical antenna is not (clearly) specified and must be accounted for. Balun unbalance is identified as an unacceptable source of error in [41], Sec. 4.

In [29] the uncertainty related to the lack of antenna height scanning is evaluated as $1.58 \mathrm{~dB}$, but it is underlined that this deviation is strongly frequency dependent. The maximum electric field value for horizontal polarization over the 1-4 m height interval is calculated for two distances (10 m and $30 \mathrm{~m}$ ) with a perfect metal ground plane and an earth plane (characterized by relative permittivity $=15$ and electrical conductivity $=0.01 \mathrm{~S} / \mathrm{m}$ ) [36]: the difference stays within $-1.4 /+0.9 \mathrm{~dB}$ over the $[30 \mathrm{MHz}$ - 
$1000 \mathrm{MHz}$ ] frequency interval. The same issue is put forward in [37]: height scanning is necessary to reduce the errors introduced by the presence of a ground plane, with the variability of electric field reading as a function of height as high as $15 \mathrm{~dB}$, hence the importance of avoiding the use of a reflective ground plane (which is mandatory by the way for high frequency, above $30 \mathrm{MHz}$, radiated emissions measurements as per several standards [12][17]). To uphold this statement, we must admit that first, height scanning is complex and time consuming and second, a reflective ground plane is not available for the in situ measurement of emissions from large machinery and apparatus, which are not covered by the above cited standards. Alexander [38] underlines that fixed antenna heights are potentially the more accurate, but more than one height may be required to cover nulls. Also Smith [39] states that fixed height method eliminates the dependence of results on antenna parameters at the expense of "exaggerating” differences between sites.

Coming back to how Antenna Factor is derived [30], with ANSI C63.5 taken as reference, not only some error terms due to mutual coupling, large antenna physical dimensions, electrical center position and not perfect (deviating from $\sin \theta$ ) radiation patterns must be corrected [30], but also it must be underlined that the calibration method is suitable for height scanning with a point source exactly at the assumed position for calibration setup, $2 \mathrm{~m}$ height, which is not the case for large power machinery. So two additional sources of errors (and related uncertainty) must be considered: first, for small variations of height with respect to the $2 \mathrm{~m}$ reference value, we may treat the issue as for antenna electrical center variations, but larger variations may introduce errors as high as those identified in [37] for height scanning $(<15 \mathrm{~dB})$; second, the source of emissions is not a point source and this is a quite complicated subject, with not only multiple paths of emissions and related reflections, but also a not well defined source position (and related height), which may be represented only by an average height (for which we may apply a correction factor) and uncertainty associated to height spanning. A second order additional uncertainty term is represented for the lowest frequencies and, as a consequence, for the longest dipole elements, by the droop of several $\mathrm{cm}$ under their own weight [42]: the related uncertainty is only $0.03 \mathrm{~dB}$ with rectangular distribution. Not to mention that whenever farfield assumption and related formulas are used to derive AF, the error below around $50 \mathrm{MHz}$ for not Hertzian antennas is not negligible: the effective distance $r$ may be related to the spatial distance $d$ by the formula [30], that includes field reactive terms,

$$
r=\frac{d}{\sqrt{1-\frac{1}{(\beta d)^{2}}+\frac{1}{(\beta d)^{4}}}}
$$

with $\beta=\lambda / 2 \pi$, which is closely related to the complete E-field expression for a Hertzian dipole including reactive field terms with linear and quadratic behavior with distance. If one fails to do so, in the restricted frequency interval between 30 and $50 \mathrm{MHz}$ approximately, the error is about $1 \mathrm{~dB}$ at 40 $\mathrm{MHz}, 4 \mathrm{~dB}$ at $30 \mathrm{MHz}$ and $7 \mathrm{~dB}$ at $20 \mathrm{MHz}$, as calculated by Alexander [30] for AFs determined with SAE ARP 958 method. True antenna separation is strongly related, especially for $3 \mathrm{~m}$ and closer 
distances, to antenna orientation (think of biconical antennas and antenna tip), that in turn is related to Horizontal and Vertical polarization (HP and VP), for which different coupling to ground plane must be accounted for. In author's opinion this is a very troublesome issue, since figures of site uncertainty for HP and VP may be implicitly also related to antenna separation during calibration and not only to electric phenomena concerning coupling.

Numerical values associated with the error sources and uncertainty terms considered above are given in Table IV. Reference is made mainly to [29] and [42]: while the former represents a high quality and realistic uncertainty budget, the latter, where a National metrological laboratory is used as reference, indicate the lowest uncertainty figures attainable. For some terms reference is made also to [30] and to [32], Table III. If the numbers drawn from [29] are considered, then a total expanded uncertainty $(k=2)$ of approximately $\pm 2 / 3 \mathrm{~dB}$ is obtained, to be compared with the reference limit represented by the results of [42] of only about $\pm 0.15 \mathrm{~dB}$.

Moreover, compliant OATS represent only a fraction of all test sites: a significant fraction of all the OATS, SAC and FAC that are formally compliant [27]-[29], but only a small fraction if test rooms and test benches (used also for non-EMC tests) at manufacturers' workshops are included [36]; so, a generic test site, where error sources are not completely known nor under control by means of adequate correction factors, exhibits a much larger uncertainty. As a last example, as underlined in [39] for the results concerning site attenuation of a large indoor factory site, an additional significant error term (that makes calculated site attenuation departing from the measured one) is the contribution of ceiling-reflected waves, that produces oscillations of measured site attenuation values, as large as $12 \mathrm{~dB}$ with respect to the theoretical curve. An attempt to quantify the uncertainty of a real site at manufacturer's workshop is done in Table V.

After all the information and experimental data reported above, it must be underlined that to author's knowledge there are no equivalent procedures and technical references for site compliance evaluation and estimation of error sources and related uncertainty in the low frequency range $[9 \mathrm{kHz}-$ $30 \mathrm{MHz}]$. It must be remembered that all measurements in this frequency range occur in the near-field region, so that theoretical computation of site characteristics is very difficult.

For the considerations on antenna mutual coupling and coupling to ground, where the largest uncertainty was for the lowest frequencies, the use of small antennas is advisable, such as magnetic and electric field probes (shielded loops and a series of electrical dipole geometries, such as cylindrical dipole, hollow spherical dipole, base-loaded monopole over a ground plane or conical monopole with hemispherical cap over a ground plane): several error sources concerning antenna coupling and its influence on Antenna Factor may be neglected.

If reflection and interaction of the emission source with conductive surfaces are considered, the presence of significant magnetic field emissions at low frequency (indicated by low calculated wave impedance values), suggests that magnetic diffusion and penetration inside solids are of concern too. 
Missing a standard procedure equivalent to that for NSA determination, a generic test site may be characterized for the low frequency interval in a similar way with attention to: a) reflections and reflection rays (as for the rationale for CISPR elliptic obstruction free area); b) polarization and height above ground, including mutual coupling effects; c) sensitivity to the source position with respect to the ambient, not only to tackle unwanted reflections and interactions, but also to form a database for the interpretation of emission measurements from large dimensions sources; d) avoid height scanning, which leads to several errors due to the absence of a perfectly reflecting ground plane and a suitable obstruction free area and errors in the determination of the antenna-EUT distance for non-point sources.

It becomes evident that site uncertainty is the largest and most influent factor in the overall test uncertainty budget, where "site uncertainty" includes a variety of error sources related also to antenna calibration methods, their usage and measurement procedures. Electromagnetic mapping of the test site and of external sources and consistency evaluation of recordings are then two important aspects for the correct measurement of e.m. emissions from large power machinery.

\section{Measurement procedure and post-processing methods}

Based also on the discussion above for site NSA determination, a procedure (consisting of five steps) is proposed for antenna placing, execution of measurements and post-processing of measurement results.

1) Antenna positions with respect to the EUT are chosen so that: a) no metallic or conductive objects are interposed; b) the signal-to-noise ratio (estimated with sample preliminary measurements performed on a grid of selected positions) is adequate. This is the advice given also in [21], where reduction of measurement distance below the $3 \mathrm{~m}$ standard is proposed to increase EUT signal contribution.

2) Several recordings are performed and corrupted recordings are recognized (and discarded) by a preliminary heuristic analysis, aimed to detect measurement mistakes and intermittent, anomalous noise from external sources, that cannot be fit within the statistical analysis outlined in section 2. At least $n$ valid recordings are performed for a) each measuring position, b) E or $\mathrm{H}$ field measurement, c) antenna orientation and d) frequency range. Analysis by means of Student's non central $t$-distribution is proposed in EN 55013 [19] in the section "Compliance with limits on a statistical basis"; as a comparison with normal distribution (for which $k=1.96 \cong 2$ ) for a confidence level of $95 \% k$ goes through the following values $(2.78,2.57,2.45,2.23)$ for $n=5,6,7,11$. For $n=5$ the coverage factor is increase by $41 \%$.

3) Uncertainty terms, as defined in section 3, are evaluated for a) the instrumentation (Type B, based on manufacturer's data and calibration service), b) external sources and, in general, repeatability 
(Type A, with clearly identified algorithms for calculation of statistical properties), c) the test site, possibly by means of direct measurement (only once and then, after major site modifications).

4) Measurement results are displayed with adequate numerical and graphical means (average values, peak envelope as a worst case, more complex statistical representations), together with total measurement uncertainty and ambient noise level. A model of machine emissions may be included to fit measurement results.

5) Comparison with limits is possible only for the high frequency range [30MHz-1GHz] [3]-[5]. For comparison measured values are transformed to $10 \mathrm{~m}$ or $30 \mathrm{~m}$ distance equivalent values, but with an inverse linear distance extrapolation factor, that however is only valid for free space conditions [43].

\section{Sample measurements and results}

Type B uncertainties for the instrumentation used in the measurement campaigns performed at Ansaldo Generators Test Room have been calculated and the results are shown in Table II.

Test room noise may be extremely variable as it is evident from Fig. 2. At specific frequencies the (Type A calculated) $k=2$ uncertainty is very large (10 dB for some components). Results are summarized in Table III for $\mathrm{E}$ and $\mathrm{H}$ field test room noise up to $200 \mathrm{MHz}$.

External sources (like radio broadcast emitters, radio amateurs emitters, static converters and drives, lighting system, etc) must be continuously monitored as explained in section 3. First, their emissions must be measured in the absence of emissions from the machine under test and characterized in terms of amplitude and band occupation, so that they can be recognized a posteriori, especially when the sources cannot be controlled.

Second, if the sources are partially or completely under control their emissions must be eliminated (by turning the sources off) or reduced to acceptable levels (by means of adequate filters/shields). As an example the recording of electric field emissions at the startup of the lubricating auxiliary system (while the receiver was scanning $58 \mathrm{kHz}$ ) is shown in Fig. 6; the increase of ambient noise is larger 10 $\mathrm{dB}$ above average ambient noise. Another example of external source is represented by a periodic spectra (shown in Fig. 1 for an E field recording at high frequency), which in our test case is very likely a static converter with a commutation frequency of about $60 \mathrm{kHz}$. This kind of large bandwidth complex emissions must be controlled and eliminated at the setup and recording level (before the storing of measured spectra takes place) not at the post-processing level.

Two series of measurements performed for no-load and short circuit conditions on the synchronous generator G1 of Table I are shown in Fig. 7 together with e.m. noise. It is evident that the generator under test is not a significant source of E field emissions, which are comparable with the ambient noise. On the contrary $\mathrm{H}$ field emission level is higher than the ambient noise up to about $4 \mathrm{MHz}$ (with 
some exceptions around $3 \mathrm{MHz}$ ), with a signal-to-noise ratio well above $10 \mathrm{~dB}$. In the $\mathrm{H}$ field spectra the $1.3 \mathrm{MHz}$ component due to radio broadcast transmissions is clearly visible.

The comparison of $\mathrm{E}$ field and $\mathrm{H}$ field emissions from synchronous generators to the test room noise suggests that the frequency range where the signal-to-noise ratio exceeds $10 \mathrm{~dB}$ is limited to nearly $5 \mathrm{MHz}$ and $1 \mathrm{MHz}$ for $\mathrm{H}$ and $\mathrm{E}$ field measurement respectively.

E field measurements above $30 \mathrm{MHz}$ confirm that there is no appreciable increase of E field intensity above ambient noise for several synchronous generators; on the contrary some measurements made on a dc motor showed that in that case E field is significant up to about $4 \mathrm{MHz}$.

For the choice of the measuring positions the goal of the tests must be clearly defined. If the EMC assessment is of concern, then a comprehensive mapping of the e.m. emissions in the space around the machine must be performed, irrespective of the best measuring point. Otherwise, if the attention is on the analysis of the e.m. behavior of the machine and its correlation with machine variables, then the signal-to-noise ratio is an important factor for the quality and interpretation of results. Several factors have been considered specifically for the GTR site: the amount of e.m. noise produced by the test room apparatus; the characteristics of the e.m. noise produced by the dc motor driving the generator; the attenuation with distance of the e.m. emissions of the generator; the characteristics of the emissions produced by the generator, which are much higher away from the machine axis (radial positions).

It may be stated that good measuring positions are: away from the generator axis, on the opposite side of the driving dc motor, far from the cable way carrying the cables connecting the rectifier and the field winding, only a few meters away from the source (the $10 \mathrm{~m}$ distance suggested by EMC standards is not applicable here and, in general, when performing measurements within a workshop environment with high levels of e.m. noise).

By inspection of Fig. 8 it may be concluded that the higher magnetic emissions are measured for the loop angles of $90^{\circ}$ and $135^{\circ}$ with respect to machine longitudinal axis. The explanation is that the $\mathrm{H}$ field lines originate from the machine ends on the machine axis and close around in the longitudinal direction (with respect to machine axis). For this machine the magnetic field intensity in the vertical axis is lower than that in the horizontal plane. This is not a general rule, but it's strongly related to machine structure and operation; tests performed in the same period on a brushless synchronous generator showed a significant increment of magnetic field intensity in the vertical axis [8], which indicated that other $\mathrm{H}$-field lines close in radial direction (orthogonal to machine axis).

Moreover, the higher level of the magnetic emissions have been recorded for the no load test and not for the short circuit test; on the contrary, for the brushless generator [8] the magnetic emissions in the horizontal plane are similar for the two tests, while the magnetic field in the vertical axis shows an increment of about $15 \mathrm{~dB}$ during short circuit tests.

Hence, the best antenna orientation and the type of test (no load, short circuit, other) for recording the highest magnetic emissions depend on the specific machine; based on author's experience, the 
correlation of the magnetic emissions with the machine characteristics is a very difficult task, so that the choice of the operating conditions for maximum emissions must be done on the field, exploring at least no load and short circuit conditions.

\section{Conclusions}

A measurement procedure, together with uncertainty evaluation, has been presented for e.m. emissions from large power rotating machinery have been defined and demonstrated with the help of some test cases.

Uncertainty related to measurement instruments, external noise sources and test site has been considered, with the indication of average values of general validity and specific values for the equipment used during the measurement campaigns at Ansaldo Generator Test Room.

Some considerations have been done on peculiar large power machinery characteristics and on the physical phenomena involved while evaluating machine emissions. The frequency range where $\mathrm{E}$ and $\mathrm{H}$ field emissions are above ambient noise has been determined up to a few $\mathrm{MHz}$; only for collector machines measurement may be performed up to nearly $10 \mathrm{MHz}$.

\section{Acknowledgements}

Special acknowledgements to Mr. Vittorio Barbero, Mr. Antonio Motta and all the personnel working in the Generators Test Room at Ansaldo Energia S.p.A. (Genova) for the assistance during the tests.

\section{References}

[1] M. Payn and M.F. Fekete “Adjustable-speed drives’ passport to Europe: the CE mark”, Power Transmission Design, April 1996, pp. 25-27.

[2] Directive EMC 89/336/EEC on the approximation of the laws of the Member States relating to electromagnetic compatibility

[3] EN 61800-3/A11, Adjustable speed electrical power drive systems - Part 3: EMC product standard including specific test methods, 2000-10

[4] EN 61000-6-2, Electromagnetic compatibility - Generic emission standard - Part 2: Industrial environment, 1993-08

[5] EN 61000-6-3, Electromagnetic compatibility - Generic immunity standard - Part 2: Industrial environment, 1995-03

[6] R.A. Errath "EMC on AC motors - advice, tips and hints", 39th IEEE Cement Industry Technical Conference 1997, Apr 20-24 1997, pp. 39-61.

[7] N.J. Ryan et al. "Electromagnetic compatibility of electrical machines and drive systems", 30th University Power Engineering Conference UPEC 1995, Sept 5-7 1995, v. 1, pp. 141-144.

[8] P. Ferrari, A. Mariscotti, A. Motta, P. Pozzobon, "Electromagnetic Emissions from Electrical Rotating Machinery”, IEEE Transactions on Energy Conversion, vol. 16 n. 1, March 2001, pp. 68-73.

[9] A. Crane and T.J. McCoy "Electromagnetic compatibility design for a 19MW PWM motor drive", 34th IEEE IAS Annual Meeting, 1999, v. 3, pp. 1590-1595.

[10]IEC 60034-1/A2, Rotating electrical machines - Part 1: Rating and performance, 1997-06

[11]EN 60034-1, Rotating electrical machines - Part 1: Rating and performance, 2000-02

[12]EN 55011, Limits and methods of measurement of radio disturbance characteristic of industrial, scientific and medical (ISM) radio-frequency equipment, 1991-03 
[13]G. Betta, D. Capriglione and G. Tomasso, "Evaluation of the Measurement Uncertainties in the Conducted Emissions from Adjustable Speed Eletcrical Power Drive Systems”, IEEE Trans. on Instrumentation and Measurement, vol. 53 n. 4, Aug. 2004, pp. 963-968.

[14]IEEE Std. 473 “Recommended Practice for an Electromagnetic Site Survey (10 kHz to $40 \mathrm{GHz}$ )”, WG473 internal draft

[15]MIL Std. 462D “Measurement of electromagnetic characteristics”, Jan. 11, 1993.

[16]C.R. Suriano, J.R. Suriano, G. Thiele and T.W. Holmes, "Prediction of Radiated Emissions From DC Motors”, International Symp. on EMC, vol. 01.2, Aug. 24-28, 1998, Colorado, USA.

[17]CISPR 16, Specification for radio disturbance and immunity measuring apparatus and methods, IEC 1st edition, 1993-08

[18]PrEN 50121-2, Electromagnetic compatibility - Part 2: Emissions of the whole railway system to the outside world, 1999-10

[19]EN 55013, Limits and methods of measurement of radio disturbance characteristics of broadcast receivers and associated equipment, 1990

[20]K.N. Sakthivel, S.K. Das and K.R. Kini "Uncertainty in radio frequency emission measurements due to the effects of electromagnetic ambient”, IEEE International Symposium on Electromagnetic Compatibility, Vol. 2, 13-17 Aug. 2001, pp. 985-990.

[21]J. Svacina, “Open Area test Site: dealing with ambient”, Compliance Engineering, Jan. 2005.

[22]Guide to the Expression of Uncertainty in Measurement, International Standardization Organization, Geneva, Switzerland, 1993.

[23]E. L. Bronaugh and J. D. M. Osburn, "A process for the analysis of the physics of measurement and determination of measurement uncertainty in EMC test procedures”, IEEE International Symposium on Electromagnetic Compatibility, Santa Clara, CA, Aug. 1996, pp. 245-249.

[24]E. R. Heise and R. E. W. Heise, “A method to calculate uncertainty of radiated measurements", IEEE International Symposium on Electromagnetic Compatibility, Austin, TX, Aug. 1997, pp. 359-364.

[25]Tektronix, “2754/2754P spectrum analyzer - Operators manual”, rev. Oct. 1986

[26]ANSI C63.4, American National Standards for Methods of Measurement of Radio-Noise Emissions from Low-Voltage Electrical and Electronic Equipment in the range of $9 \mathrm{kHz}$ to $40 \mathrm{GHz}, 1992$.

[27]A. Crumm and K. Hall, "An update on comparison of site-to-site measurement reproducibility using HP site reference source”, IEEE International Symposium on Electromagnetic Compatibility, Minneapolis (MN), Aug. 2002, pp. 35-38.

[28]J.J. Goedbloed and P.A. Beechman, "Uncertainty analysis of the CISPR/A radiated emission Round-Robin test results”, IEEE Trans. on Electromagnetic Compatibility, vol. 46 n. 2, May 2004, pp. 246-260.

[29]T.W. Kang and H.T. Kim, "Reproducibility and uncertainty in radiated emission measurements at Open Area Test Sites and in Semianechoic Chambers using a spherical dipole radiator”, IEEE Trans. on Electromagnetic Compatibility, vol. 43 n. 4, Nov. 2001, pp. 677-685.

[30]M.J. Alexander, “Antenna calibration and the measurement of E-field strength”, Compliance Engineering, Jan. 2006.

[31]M.D. Foegelle, “Site validation theory 101: techniques and methods”, Compliance Engineering, July 2000.

[32]Z. Chen and M. Windler, "Systematic errors in normalized site attenuation testing”, Compliance Engineering, Jan. 2000.

[33]J.D. Gavenda, "Near field corrections to site attenuation”, IEEE Trans. on Electromagnetic Compatibility, vol. 36 n. 3, Aug. 1994, pp. 213-220.

[34]Z. Chen and M.D. Foegelle, “A Numerical Investigation of Ground Plane Effects on Biconical Antenna Factor”, IEEE Intern. Symp. on Electromagnetic Compatibility, Denver, CO, 1998, pp. 802-806.

[35]Z. Chen, M.D. Foegelle, and T. Harrington, "Analysis of Log Periodic Dipole Array Antennas for Site Validation and Radiated Emissions Testing”, IEEE Intern. Symp. on Electromagnetic Compatibility, Seattle, WA, 1999, pp. 618-623.

[36]A.A. Smith Jr., "Standard-Site Method for Determining Antenna Factors", IEEE Transactions on Electromagnetic Compatibility, vol. 24 n. 3, Aug. 1982, pp. 316-322.

[37]D. Mawdsley, "Measuring EMC emissions in the real world", IEEE Intern. Symp. on Electromagnetic Compatibility, Seattle, WA, 1999, pp. 613-617.

[38]M.J. Alexander, “EMC antenna considerations”, Compliance Engineering, July 2003.

[39]A.A. Smith Jr., "Calculation of site attenuation from Antenna Factors", IEEE Transactions on Electromagnetic Compatibility, vol. 24 n. 3, Aug. 1982, pp. 301-315.

[40]L. Turnbull and A.C. Marvin, "Effects of cross-polar coupling on open area test site measurement correlation and repeatability”, IEE Proceedings_Science, Measurement and Technology, vol. 143 n. 4, 1996, pp. 202-207.

[41]M.J. Alexander, "European Intercomparison of Antenna Factors in the Frequency Range $30 \mathrm{MHz}$ to $1 \mathrm{GHz}$ ”, IEE Proceedings—Science, Measurement and Technology, vol. 143 n. 4, 1996, pp. 229-240. 
[42]M.J. Alexander and M.J. Salter, "Low measurement uncertainties in the frequency range $30 \mathrm{MHz}$ to $1 \mathrm{GHz}$ using a calculable standard dipole antenna and national reference ground plane”, IEE Proceedings-Science, Measurement and Technology, vol. 143 n. 4, 1996, pp. 221-228.

[43]F. Gisin and Z. Pantic-Tanner, "Analysis of the measurement uncertainty associated with 1/R extrapolation of radiated emission measurements on an Open Area Test Site", IEEE International Symposium on Electromagnetic Compatibility, Denver, CO, August 24-28, 1998, pp. 137-140.

[44]F Gisin, "Using ANSI C63.5 Standard Site Method Antenna Factors for Verifying ANSI C63.4 Site Attenuation Requirements, IEEE International Symposium on Electromagnetic Compatibility, Dallas, TX, August 9-13, 1993, pp. 313-314. 


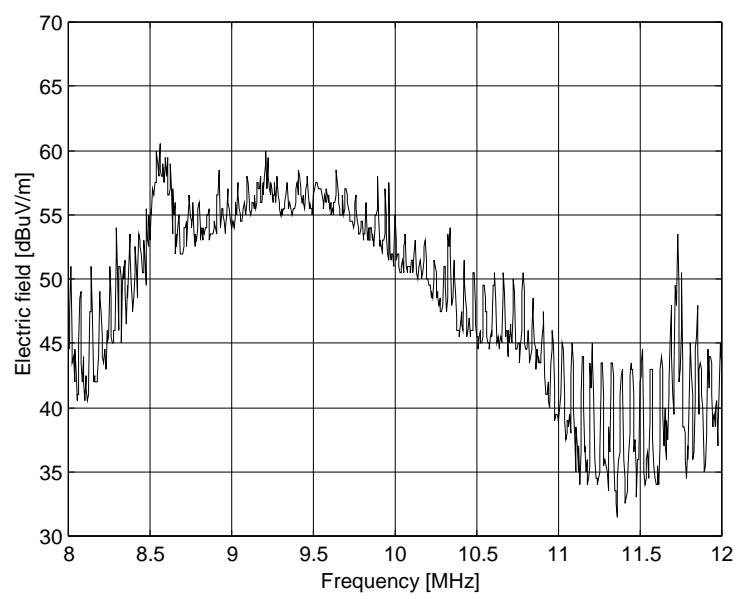

Figure 1. Electric field noise at high frequency (evidence of a "periodic" source) 

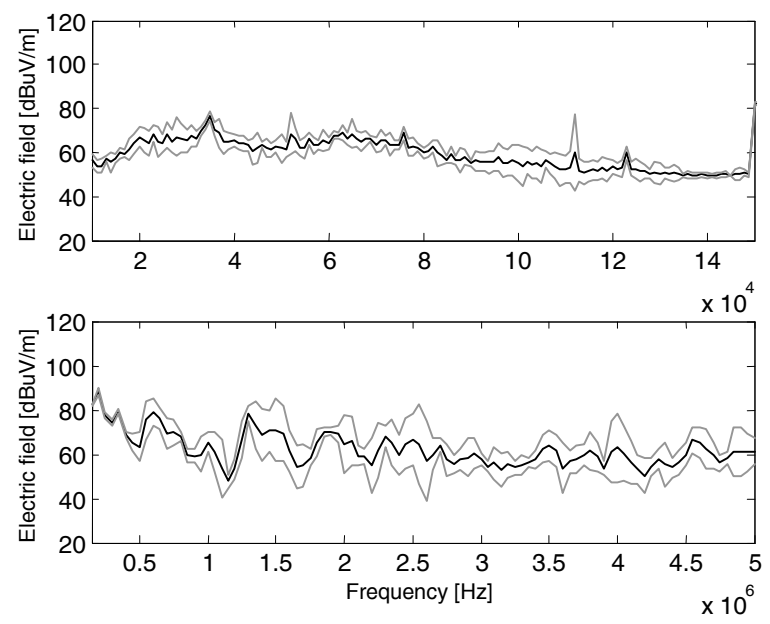

(a)
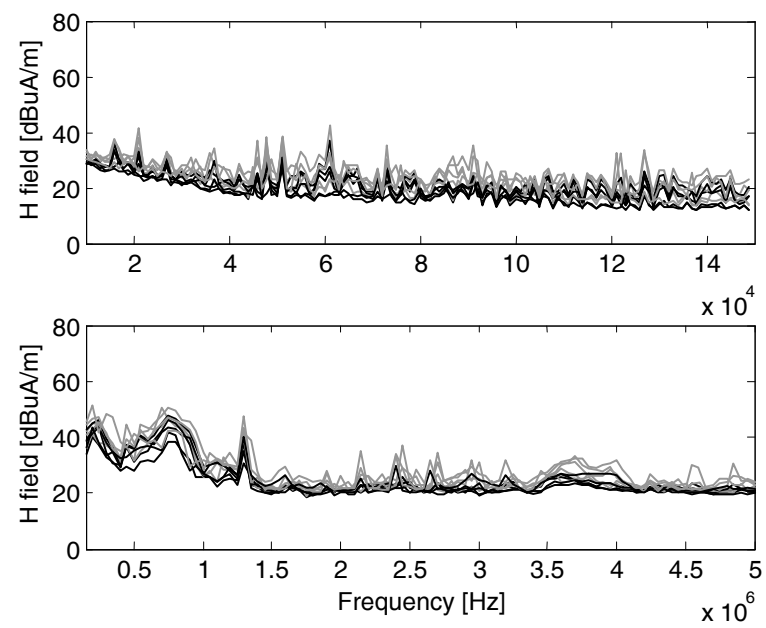

(b)

Figure 2. Test room noise (average values, black, and 95\% confidence interval, grey): (a) E-field (vertical) and (b) $\mathrm{H}$-field (4 orientation in the horizontal plane at $0^{\circ}, 45^{\circ}, 90^{\circ}$ and $135^{\circ}$ and vertical) in $\mathrm{Z} 2$

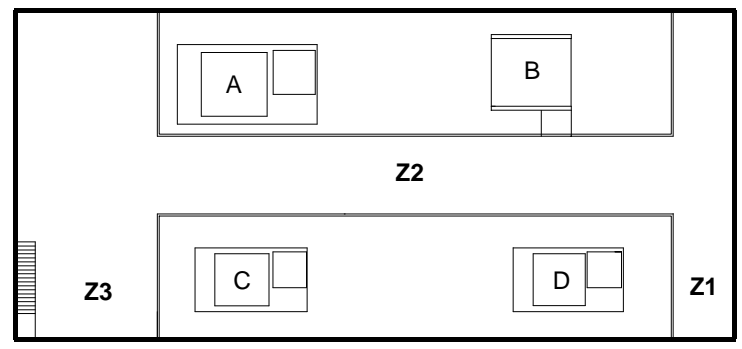

Figure 3. Map of the Generator Test Room 


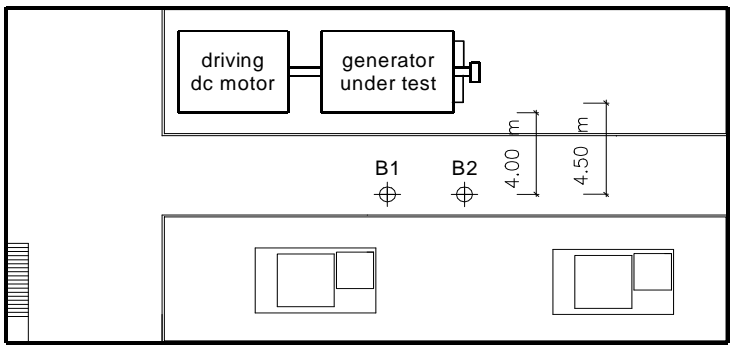

Figure 4. Placement of the synchronous generator

TABLE I. MAIN CHARACTERISTICS OF THE SYNCHRONOUS GENERATOR UNDER TEST

\begin{tabular}{|l|c|c|}
\hline Generator parameters & G1 & G2 \\
\hline Rated power [MVA] & 205 & 170 \\
Rated stator voltage [kV] & 15 & 15.7 \\
Rated stator current [A] & 7890 & 6232 \\
Rated field voltage [V] & 295 & 261 \\
Rated field current [A] & 1361 & 1246 \\
Rated speed [rpm] & 3000 & 3000 \\
Rated frequency [Hz] & 50 & 50 \\
\hline
\end{tabular}

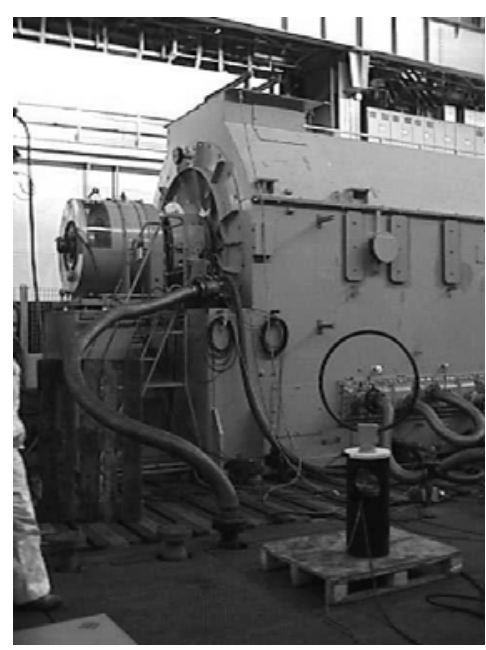

Figure 5. Picture of the synchronous generator G1 under test 
TABLE II. CALCULATED INSTRUMENTATION UNCERTAINTY

\begin{tabular}{|l|c|c|}
\hline Instrument & Distr. & Uncert. \\
\hline Antenna (loop EMCO 6502) & & \\
Antenna Factor & rect. & 0.87 \\
Input VSWR* & U-shape & $<2.5 / 1.1$ \\
Antenna (rod EMCO 3301) & rect. & 0.87 \\
Antenna Factor & U-shape & $<2.5 / 1.1$ \\
Input VSWR* & & \\
Antenna (bi-conical PMM) & rect. & 0.87 \\
Antenna Factor & U-shape & $<4.2 / 2.5$ \\
Input VSWR* & rect. & 1.75 \\
Spectrum analyzer (TEK 2754P) & 0.29 \\
Input attenuator & U-shape & 1.61 \\
Input VSWR & rect. & 0.23 \\
Gain variation & rect. & 0.58 \\
Scale interpolation & \multicolumn{2}{|c|}{} \\
\hline
\end{tabular}

* 1st/2nd values: worst case value for unmatched antenna input impedance (no attenuator), estimated value for $10 \mathrm{~dB}$ attenuator (or better).

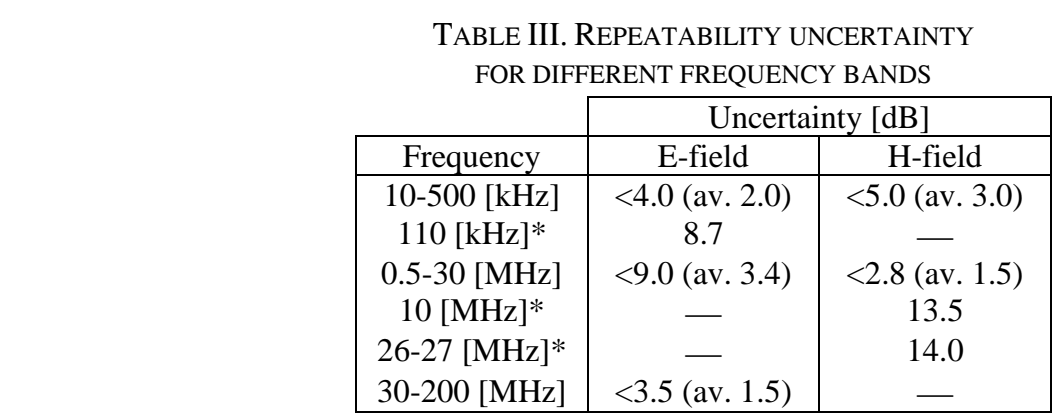

* radio broadcast frequencies 
TABLE IV. TEST SITE ATTENUATION AND ANTENNA CALIBRATION UNCERTAINTY

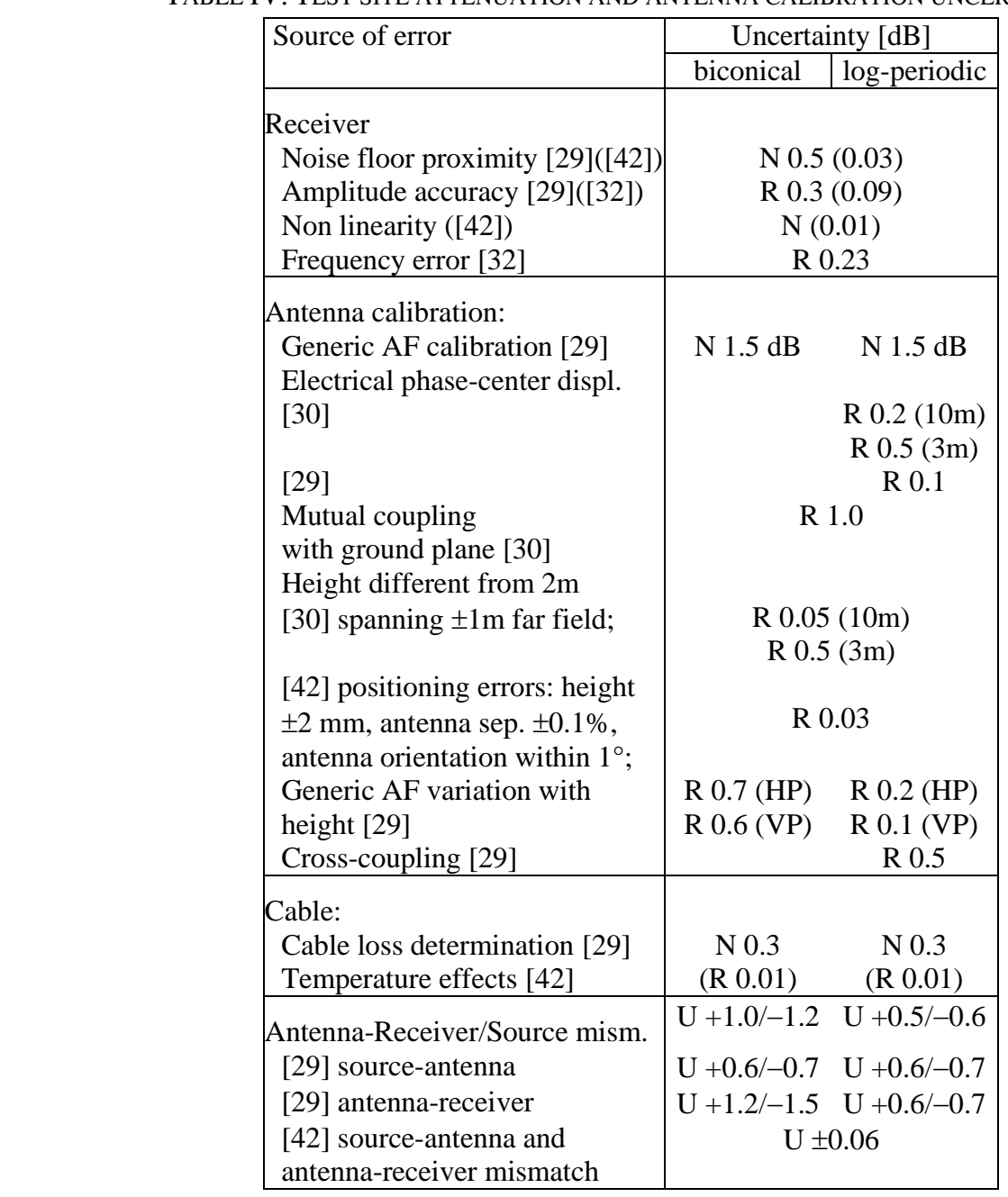

$\mathrm{R}=$ rectangular, $\mathrm{N}=$ normal, $\mathrm{T}=$ triangular, $\mathrm{U}=\mathrm{U}$-shaped.

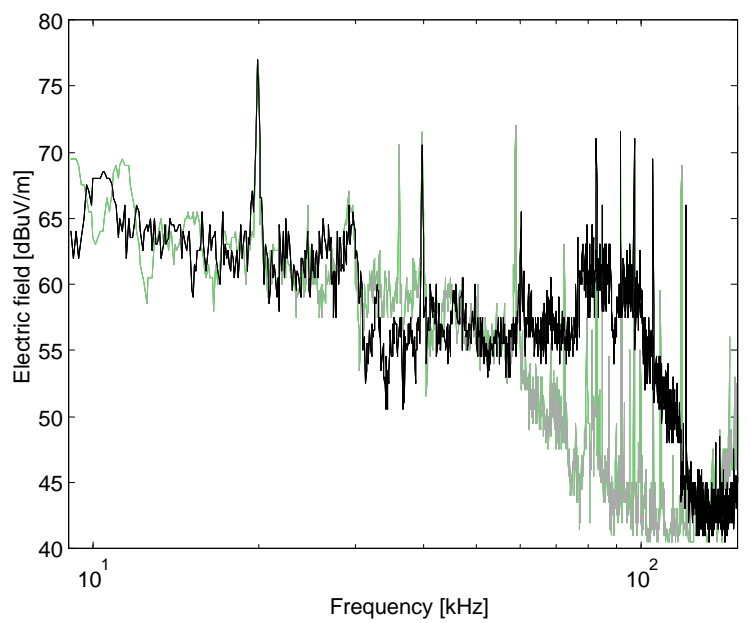

Figure 6. Electric field noise at the startup of the lubricating auxiliary system 

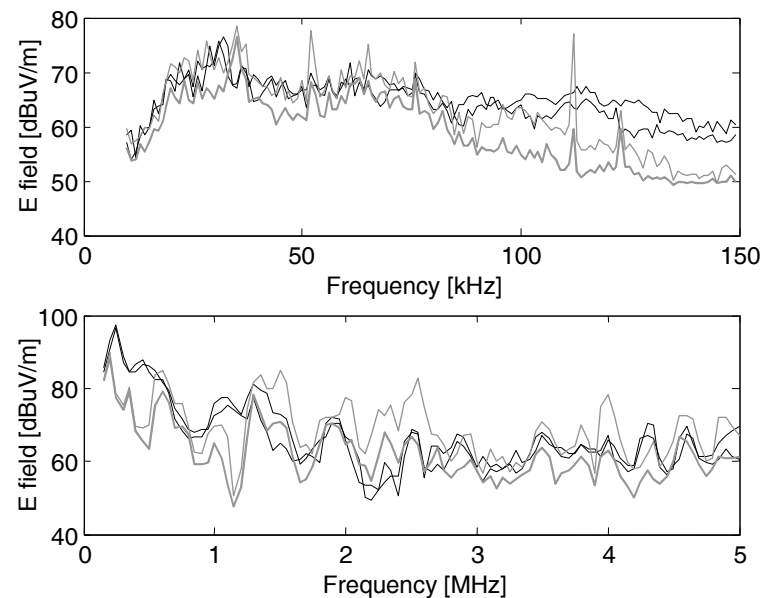

(a)
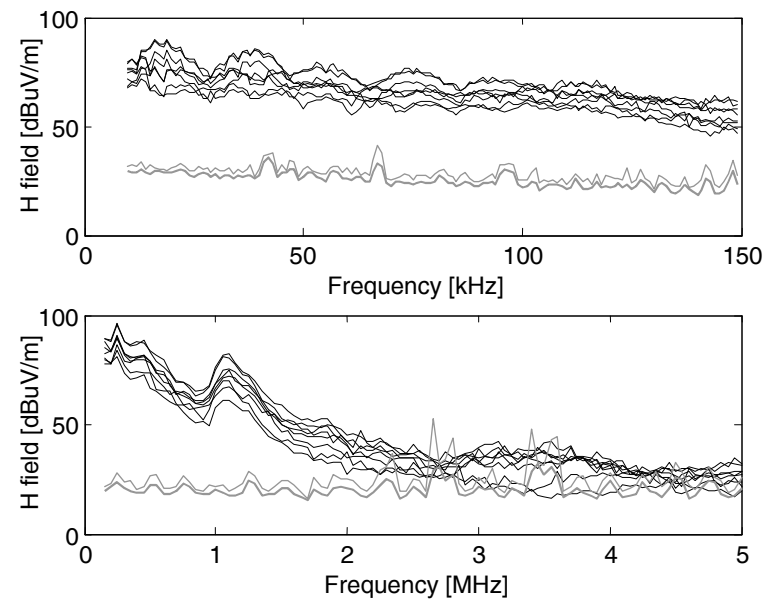

(b)

Figure 7. Comparison of machine emissions (black, for all orientations) and test room noise (grey: average value, thick, 95\% confidence interval, thin): (a) E- and (b) H-field 

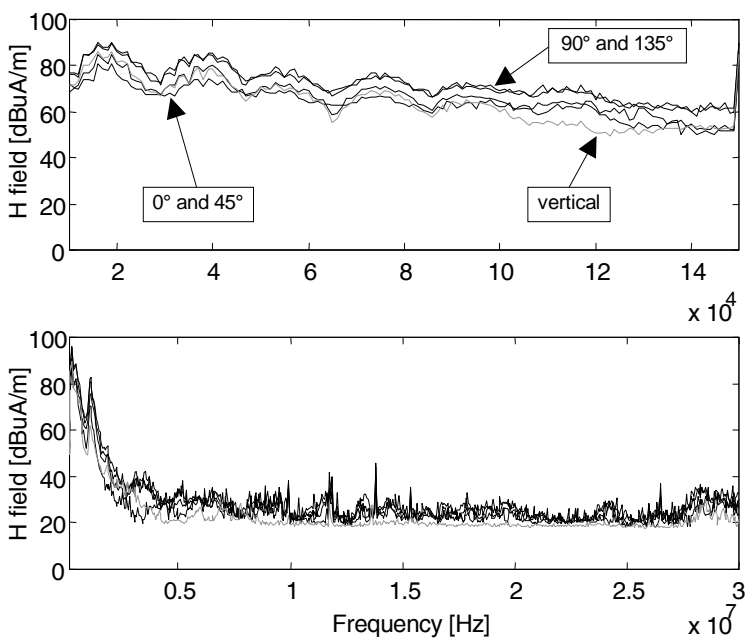

Figure 8. Example set of $\mathrm{H}$-field spectra (average value) of G1 during no-load test for different loop antenna orientations 